\title{
Plant nutrient quality impacts survival and reproductive fitness of the dengue vector Aedes aegypti
}

\author{
Vincent Odhiambo Nyasembe ${ }^{1,3^{*}}$ (D) David Poumo Tchouassi ${ }^{1}$, Martha Njeri Muturi ${ }^{2}$, Christian W. W. Pirk ${ }^{3}$,
} Catherine L. Sole ${ }^{3}$ and Baldwyn Torto ${ }^{1,3}$

\begin{abstract}
Background: In a recent study using DNA barcoding, we identified the plants fed upon by four Afro-tropical mosquito species that vector dengue, malaria, and Rift Valley fever. Herein, we have expanded on this study by investigating the role of three of the plants, Pithecellobium dulce (Fabaceae), Leonotis nepetifolia (Lamiaceae), and Opuntia ficus-indica (Cactaceae), on the survival, fecundity, and egg viability of the dengue vector Aedes aegypti.

Methods: We tested these effects using females that received (i) an initial three rations of blood meals and (ii) no blood meal at all. Two controls were included: age-matched females fed on glucose solution with or without an initial blood meal and those fed exclusively on blood meals. Data were collected daily over a 30-day period. The amino acid contents of Ae. aegypti guts and their respective diets were detected by coupled liquid chromatography-mass spectrometry.

Results: Females fed on P. dulce and an exclusively blood meal diet had a shorter survival than those fed on glucose. On the other hand, females fed on L. nepetifolia survived longer than those fed exclusively on blood meals, whereas those fed on O. ficus-indica had the shortest survival time. With an initial blood meal, females fed on L. nepetifolia laid 1.6-fold more eggs while those fed on the other diets laid fewer eggs compared to those fed exclusively on blood meals. Hatching rates of the eggs laid varied with the diet. Mass spectroscopic analysis of gut contents of mosquitoes exposed to the different diets showed qualitative and quantitative differences in their amino acid levels.
\end{abstract}

Conclusion: Our findings highlight the central role of plant nutrients in the reproductive fitness of dengue vectors, which may impact their disease transmission potential.

Keywords: Plant nectar, Plant sap, Survival, Fecundity, Hatching-rates, Amino acids, Dengue

\section{Background}

The last 2 decades have seen the resurgence and spread of arboviruses such as dengue, Zika, and chikungunya viruses, which are vectored by Aedes mosquitoes. Although the number of dengue cases are underreported and misclassified [1], the number of global infections is

*Correspondence: vnyasembe@gmail.com

${ }^{1}$ International Centre of Insect Physiology and Ecology (Icipe), Nairobi, Kenya

Full list of author information is available at the end of the article estimated at 390 million annually, with about $50-100$ million cases manifesting clinically $[2,3]$. The geographic expansion of dengue, which is caused by four dengue virus serotypes (DENV 1-4), has been characterized by increases in case incidence, epidemics, and super-endemicity, with more frequent severe forms of dengue $[4,5]$. The recent outbreak of Zika in South and Central America and the Caribbean region attests further to the continued geographic spread of arboviral diseases [6, 7]. Zika was first identified in Rhesus monkey in Uganda in 1947, with the first human cases detected in 1952 in Uganda

c) The Author(s) 2021. This article is licensed under a Creative Commons Attribution 4.0 International License, which permits use, sharing, adaptation, distribution and reproduction in any medium or format, as long as you give appropriate credit to the original author(s) and the source, provide a link to the Creative Commons licence, and indicate if changes were made. The images or other third party material in this article are included in the article's Creative Commons licence, unless indicated otherwise in a credit line to the material. If material is not included in the article's Creative Commons licence and your intended use is not permitted by statutory regulation or exceeds the permitted use, you will need to obtain permission directly from the copyright holder. To view a copy of this licence, visit http://creativeco mmons.org/licenses/by/4.0/. The Creative Commons Public Domain Dedication waiver (http://creativecommons.org/publicdomain/ zero/1.0/) applies to the data made available in this article, unless otherwise stated in a credit line to the data. 
and Tanzania [8]. The Zika outbreaks in Brazil and Colombia in 2015 and its subsequent spread to 13 other countries in the Americas, along with other outbreaks in the Pacific (Yap, 2007; French Polynesia, 2013) and Africa (Cape Verde, 2015), highlight the growing concern about the rapid expansion of arboviral diseases [9]. The overarching commonality among these diseases is that there are currently no specific drugs for their treatment and no viable vaccines available [10]. This makes effective vector control the mainstay for prevention and control of these diseases.

The geographic expansion of these diseases closely follows the tropical and subtropical distribution of their primary vectors, Aedes aegypti and Aedes albopictus. The spread has been attributed to a range of factors including climate change, uncontrolled urbanization, globalization, travel, trade, socioeconomics, and the ability of these viruses to evolve $[5,11]$. In addition, factors contributing to the resilience of Ae. aegypti populations such as insecticide resistance, ability of eggs to withstand desiccation, ability of adults to adapt to environmental modifications, and their behavioral plasticity have contributed to the sustenance or even expansion of Ae. aegypti populations $[12,13]$. Overall, the continued expansion of the geographic range of these mosquito species and the pathogens that they transmit calls for a detailed understanding of vector and disease ecologies in a renewed effort to develop innovative management strategies.

Plant feeding is emerging as a key ecological factor in the biology of several mosquito species including Aedes species [14-17]. While plant feeding pre-dates blood feeding in insects, blood sucking arthropods are thought to have adopted the latter trait during evolution to enhance the propagation of their progeny [18]. Among different mosquito species, intermittent plant feeding in females has long been documented but its role with respect to reproductive fitness has been downplayed by different studies [19-22]. Central to this dogma are $A e$. aegypti and Anopheles gambiae, the two highly anthropophilic and most important disease vectors. Variably low fructose levels detected in field-collected females of these species accompanied by their tendency to have multiple blood meals has led to the proposition that they seldom feed on plants but depend on human blood for both their metabolic processes and reproduction. However, recent evidence where more sensitive trapping strategies and analytical approaches were used shows higher plant feeding frequencies in these two species [16, 17, 23].

Several studies have demonstrated the central role played by plant sugars in male and female mosquito survival, mating competence, and flight activity. In addition, there has been substantial effort to identify plant species fed upon by different mosquito species. These efforts have been greatly boosted by the advent of highly sensitive analytical techniques such as plant DNA barcoding and mass spectrometry, which provide secure host plant identification and authenticate their trophic association [24, 25]. Molecular approaches have recently been used to identify plant species fed upon by important disease vectors such as An. sergentii [23], An. gambiae, Ae. mcintoshi, Ae. ochraceus and Ae. aegypti [16], and phlebotomine sand flies $[26,27]$ in their natural habitats. Evidence of more frequent plant feeding among these vectors and the identification of host plant species further augment the proposition of their central role in vector population dynamics. However, beyond a few studies linking plant feeding to mosquito survival, little is known about the nutritional contribution of plants to vector fitness and population dynamics.

Building on our recent identification of natural host plants of four Afro-tropical mosquito species [16], we sought to elucidate the role of plant nutrition using three of the identified plants on survival and reproductive fitness of Ae. aegypti.

\section{Methods}

\section{Experimental animals}

Adult Ae. aegypti obtained from eggs collected in Kilifi $\left(3.6333^{\circ} \mathrm{S}, 39.8500^{\circ} \mathrm{E}\right)$ in the coastal region of Kenya endemic for dengue (Sang and Dunster, 2001) were used. The eggs were collected by placing black ovicups lined with brown ovistrips in pre-identified A. aegypti breeding sites overnight. The collected eggs were either hatched immediately or carefully dried and transported to icipe laboratories in Nairobi. The hatching larvae were reared in plastic trays $(25 \mathrm{~cm}$ long $\times 20 \mathrm{~cm}$ wide $\times 14 \mathrm{~cm}$ high) to adults with a daily ration of Tetramin fish food (Tetramin1, Melle, Germany) of 0.3 g/100 larvae/day. The rearing room was maintained at a temperature of 28 $( \pm 1){ }^{\circ} \mathrm{C}$ and relative humidity of $80( \pm 5) \%$ and a photoperiod of 12: 12 (light: dark) hours. The haplotypes of the emerging adults were all confirmed to be Ae. aegypti aegypti (hereafter referred to as $A e$. aegypti) as they all had white scales on the first abdominal tergite (McClelland,1960). The adults, 1-2 days old with no prior exposure to any other nutrient source, were used in survival and fecundity assays.

\section{Plant materials}

Plant species identified as natural host plants of the four mosquito species from our previous study [16] were used in these assays although specific for the vectors from their respective ecologies. These included Pithecellobium dulce (Roxb.) Benth (Fabaceae; Ae. aegypti host plant), Leonotis nepetifolia (L.) R.Br (Lamiaceae; An. gambiae 
host plant) and Opuntia ficus-indca (L.) Mill (Cactaceae; Ae. mcintoshi and Ae. ochraceus host plant).

Studies with $P$. dulce were conducted at the KEMRIWellcome Trust laboratories in Kilifi Kenya $\left(3.6333^{\circ} \mathrm{S}\right.$ and $39.8500^{\circ} \mathrm{E}$ ), where both Ae. aegypti and its host plant $P$. dulce co-occur. $P$. dulce is a perennial tree reaching a height of about 10-15 m; hence, we were not able to obtain it as a potted plant. Consequently, fresh cuttings of its leaves, newly blossomed flowers and young pods were used. These were changed daily over the 30-day experimental period.

Studies with $L$. nepetifolia and O. ficus-indica were conducted at icipe in Nairobi. Wild growing L. nepetifolia (obtained from Ahero, western Kenya; $0^{\circ} 10^{\prime} \mathrm{S}, 34^{\circ} 55^{\prime} \mathrm{E}$ ) and O. ficus-indica (obtained from Ijara, northeastern Kenya; $1.5988^{\circ} \mathrm{S}$ and $40.5135^{\circ} \mathrm{E}$ ), were transplanted into pots (D $25 \times \mathrm{W} 27 \times \mathrm{H} 30 \mathrm{~cm}$ ) and transported to icipe laboratories in Nairobi. They were used when they started to blossom.

All the experiments were conducted under controlled conditions as described above for mosquito rearing.

\section{Survival, fecundity, and egg hatchability of Aedes aegypti on different host plants}

In Experiment I carried out in Kilifi, two assays were conducted. In the first assay, a group of 100 males and 100 females was introduced into a $30 \times 30 \times 100 \mathrm{~cm}$ cage containing $P$. dulce cuttings. In addition to the plant, which was continuously available, they were provided with initial three blood meals from mice at day 3, 5 and 7 from the onset of the assay (Fig. 1, Assay 1). The blood meal was provided by placing an anesthetized mouse on top of the mosquito cages and allowing the mosquitoes to feed on them for an hour. Oviposition cups were provided in all the cages $48 \mathrm{~h}$ after the first blood meal. They were monitored for survival and fecundity daily for 30 days. Mortality and the daily number of eggs laid were recorded and fresh oviposition cups provided. Control experiments comprised 100 females and 100 males of Ae. aegypti with access to (i) $6 \%$ glucose solution (the concentration routinely used in mosquitoes reared at icipe) plus three initial blood meals (Fig. 1, Assay 1) and (ii) blood meals only on alternating days for 30 days with a total of 15 blood meals. Nine living female mosquitoes were randomly selected from each replicate of all the treatments on day 15 for amino acid analysis as described below. The choice of day 15 for nutrient analysis was informed by the need to allow enough time for all blood-derived nutrients to be absorbed/cleared from the mosquito gut and increase detection of plant-derived nutrients. The second assay was the same as the one above, but no blood meal was provided (Fig. 1, Assay 2). Nine living female mosquitoes were randomly selected from each replicate of all the treatments on day 15 for amino acid and sugar analysis as described in Additional file 1. A
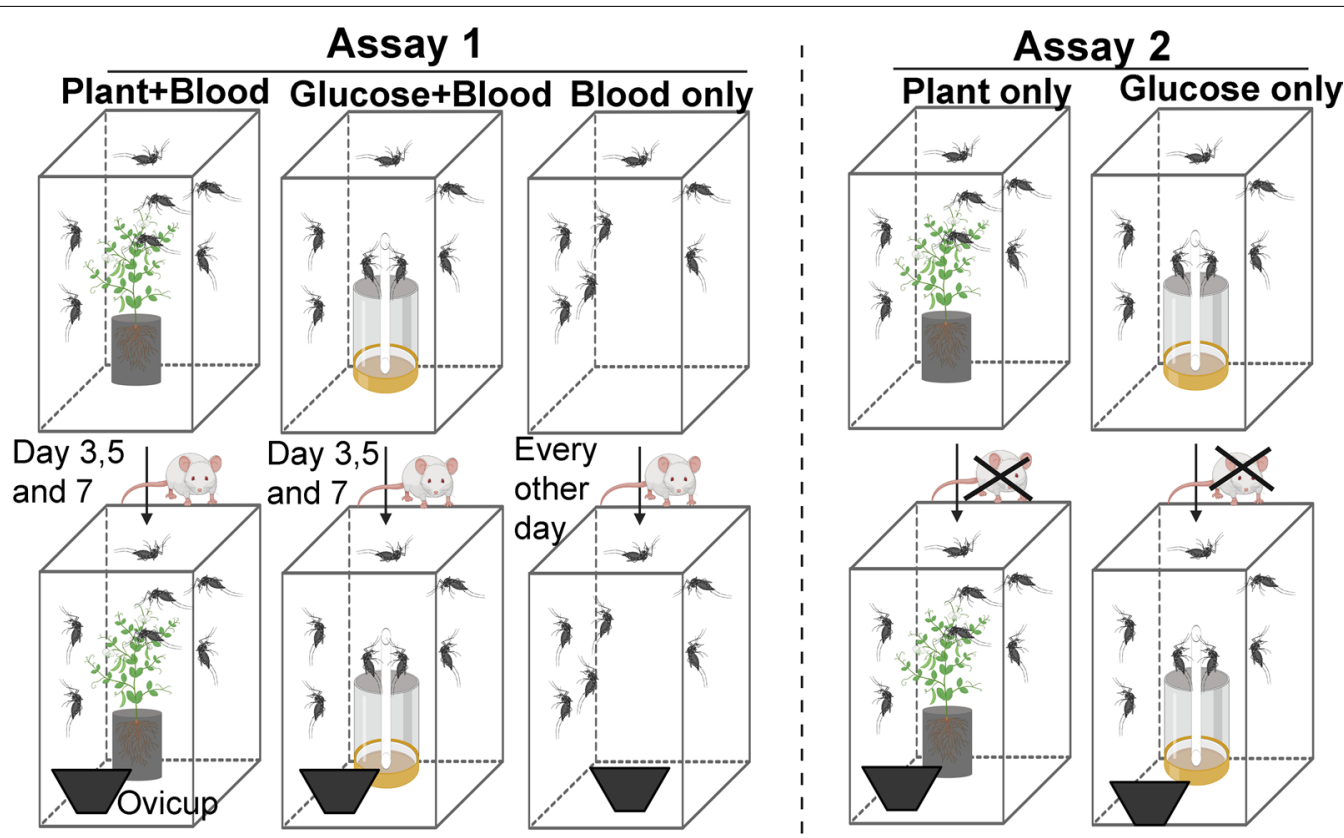

Fig. 1 Schematic representation of the study design. Mosquitoes were either fed on mouse blood (Assay 1) or no blood at all (Assay 2). Ovicups were placed in the cages on day 5 and monitored daily for eggs over the 30-day assay period; eggs were collected and the cups replaced with fresh ones every day. Sugar comprised $6 \%$ glucose solution. The images were created with BioRender.com 
total of three replicates using three different batches of mosquitoes were carried out for all nutrient regimes for both assays.

Similar experimental setup as above was used in Experiment II for survival and fecundity assays using $L$. nepetifolia and O. ficus-indica. Newly emerged females and males (100 mosquitoes for each sex) were provided with $L$. nepetifolia, O. ficus-indica, $6 \%$ glucose solution or mouse blood meal provided on alternate days. Except for the latter group, the mosquitoes in all the other three groups were either provided an initial three mouse blood meals on days 3, 5 and 7 (Fig. 1, Assay 1) or no blood meal at all (Fig. 1, Assay 2). Survival, fecundity and mosquito sampling for nutrient analysis was done as described above. The collected eggs were wrapped between moist paper towels and put in covered plastic trays for $48 \mathrm{~h}$ and then transferred to a dark container and allowed to dry slowly under rearing conditions [temperature of 28 $( \pm 1){ }^{\circ} \mathrm{C}$ and relative humidity of $\left.80( \pm 5) \%\right]$.

To measure the hatchability of the laid eggs, the eggs were put in $18 \times 12.5 \times 2.1$-inch trays and distilled water added to a depth of 1 inch. The water was first boiled and then allowed to cool to room temperature before introducing the eggs. The eggs were hatched according to the date laid and nutrient source. The number of larvae was counted daily for up to 2 weeks after which the unhatched eggs were considered not viable. The counted larvae were promptly removed.

\section{Analysis of host plant nutrient content and the ingested equivalence in Aedes aegypti}

To understand the differences in the performance of $A e$. aegypti on different nutrient sources, we quantified the amount of amino acids in the three plant species and the corresponding amounts ingested by the mosquitoes. Both plant sap from phloem in the succulent tissues and nectar from the nectaries were collected from the three plants using $20 \mu \mathrm{l}$ micro-capillary tubes (Drummond Scientific Company, Brumall, PA, USA) tapered on one end using a glass puller. Between 10 and $30 \mu \mathrm{l}$ of plant sap and nectar was separately collected, transferred into 1.5-ml low-binding Eppendorf tubes, snap frozen in liquid nitrogen and immediately stored at $-80{ }^{\circ} \mathrm{C}$ until analysis. Up to $100 \mu \mathrm{l}$ of venous blood was drawn from mouse facial veins into 1.5 -ml low-binding Eppendorf tubes and immediately snap-frozen in liquid nitrogen and stored at $-80{ }^{\circ} \mathrm{C}$. Blood was collected from three mice drawn from different litters for both experiments conducted at KEMRI-Wellcome Trust Laboratories and icipe. The mosquito samples were prepared by dissecting mosquitoes preserved from survival assays and pooling the midgut plus crop (hereafter referred to as gut) from three mosquitoes.

To detect the amino acid content, the pooled guts, $10 \mu \mathrm{l}$ of plant sap + nectar or $10 \mu \mathrm{l}$ of blood samples was hydrolyzed with $6 \mathrm{M} \mathrm{HCl}$ for $3 \mathrm{~h}$ as described by MoranPalacio et al. (2014). The product was re-suspended in $200 \mu \mathrm{lmmol} 1$ 1-1 EDTA solution, $\mathrm{pH} 7.5$ and incubated for $90 \mathrm{~min}$ in the dark in a sealed chamber equilibrated at $25{ }^{\circ} \mathrm{C}$ with a dish of saturated $\mathrm{KH}_{2} \mathrm{PO}_{4}$ to maintain high humidity. The EDTA samples were subsequently diluted in a water-acetonitrile solvent mixture in a ratio of 80:20 and analyzed on a liquid chromatograph attached to 6120 quadrupole mass spectrometer (Agilent Technologies). Samples were injected via the Agilent Technology 1260 Infinity series sample manager, injecting $10 \mu \mathrm{l}$ on to the Agilent SB-C18 $3.5 \mu \mathrm{m} 4.6 \times 250 \mathrm{~mm}$ column. The run time was $22 \mathrm{~min}$ at a flow rate of $0.7 \mathrm{ml} / \mathrm{min}$. The solvent system consisted of A (water $+1 \%$ formic acid) and B (acetonitrile $+1 \%$ formic acid). The mobile phase used a gradient program, initially $95: 5$ (A: B), to $70: 30$ at $3 \mathrm{~min}$, 20:80 at 7.5 $\mathrm{min}, 0: 100$ at $13 \mathrm{~min}$ and 95:5 at $20 \mathrm{~min}$. The mass spectrometer was operated in positive ion mode, with a capillary voltage of $3 \mathrm{kV}$, with voltage $50-180 \mathrm{eV}$ and mass range $50-300 \mathrm{~m} / \mathrm{z}$. The source temperature was $130{ }^{\circ} \mathrm{C}$, desolvation temperature $350{ }^{\circ} \mathrm{C}$, desolvation gas flow $100 \mathrm{ml} / \mathrm{min}$ (nitrogen) and con gas flow $0.7 \mathrm{ml} / \mathrm{min}$ (nitrogen). The amino acids were identified by comparing their mass spectra with the literature data [28].

The sugar contents of guts from males and non-blood fed female Ae. aegypti fed on different nutrients were analyzed as described in supplementary information (Additional file 1).

\section{Statistical analyses}

For each nutrient regime, survival data from the three replicates was first subjected to GLM to determine if they could be pooled. Since no difference was detected, data from the three replicates were pooled and the difference in survival times of adult Ae. aegypti on different nutrient sources detected using Kaplan-Meier and Cox regression survival analyses. Mosquitoes sampled for nutrient analyses and those surviving after the 30-day observation period were treated as censored. The number of eggs laid on each day was corrected to the number of surviving females, and differences in fecundity between mosquitoes held on different nutrient sources were detected using zero-inflated GLM. The differences in hatching rate from different nutrient sources were compared using one-way ANOVA and Tukey post-hoc test. The gut amino acid content was quantified for the different nutrient sources and the differences detected using one-way analysis of variance. The differences in gut sugar content was detected using ANOVA and Tukey post-hoc test. All 
statistical analyses were done in R software version 3.6.3 [29].

\section{Results}

Host plants variably support Ae. aegypti survival, fecundity and egg viability

In Experiment I, Ae. aegypti fed on glucose survived significantly longer (23.5 days) than those fed on $P$. dulce and blood (17.7 days) or blood alone (16.1 days) (log rank $=40.785, \mathrm{df}=2, P<0.001$; Fig. $2 \mathrm{a}$ ). With no initial blood meal, the mean survivals of female Ae aegypti on glucose and $P$. dulce were $23.6 \pm 0.8$ and $13.1 \pm 0.8$ days, respectively ( $\log$ rank $=48.04, \mathrm{df}=1, P<0.001$; Fig. $2 \mathrm{~b}$ ). Similar survival patterns were observed in males, with those fed on glucose having a mean survival of $21.6 \pm 0.7$ days while those fed on $P$. dulce had a median survival of $14.6 \pm 0.7$ days $(\log \operatorname{rank}=25.162, \mathrm{df}=1$, $P<0.001$; Fig. 2c).

In Experiment II, survival of Ae. aegypti females provided with an initial blood meal and fed on the different diets was significantly different (log rank $=419.727$, $\mathrm{df}=3, P$ value $<0.001$; Fig. $2 \mathrm{~d}$ ), with mean survival of $24.0 \pm 0.6,17.0 \pm 0.4,7.2 \pm 0.4$ and $16.1 \pm 0.8$ days among glucose, L. nepetifolia, O. ficus-indica and blood, respectively. With no initial blood meal, the mean survivals on glucose solution, L. nepetifolia and O. ficus-indica were $23.9 \pm 0.7,25.2 \pm 0.5$ and $7.4 \pm 0.2$ days for females ( $\log \operatorname{rank}=485.405, \mathrm{df}=2$, $P$-value $<0.001$; Fig. 2 e) and $21.0 \pm 0.6,14.5 \pm 0.5$ and $6.5 \pm 0.4$ days for males $(\log \operatorname{rank}=354.639, \mathrm{df}=2$, $P$-value $<0.001$; Fig. 2f), respectively.

For fecundity, females fed on $P$. dulce and glucose laid 1.6- and 2.2-fold fewer eggs, respectively, than those fed exclusively on blood but no significant difference was detected $\left.\left(\mathrm{F}_{(2,267}\right)=1.985, \mathrm{I}=0.139\right)$. On the other hand, those fed on L. nepetifolia laid 1.6-fold more eggs than those fed exclusively on blood, while mosquitoes fed on O. ficus-indica and glucose had 1.7- and twofold fewer eggs than those fed exclusively on blood meals, respectively $\left.\left(\mathrm{F}_{(3,356}\right)=3.495, P=0.0158\right)$. Besides having the highest fecundity rate, mosquitoes fed on L. nepetifolia had a sustained moderate oviposition throughout the experimental period which was comparable to those that exclusively fed on blood meals (Fig. 3a, b).

Regarding egg viability, marginally significant differences were detected in the median hatching rates of eggs laid by mosquitoes fed on glucose (0.10), $P$. dulce (0.0.16) and blood (0.65) $\left(\mathrm{F}_{(2,31}\right)=3.344, P=0.0484$; Fig. 4a). On the other, a significant difference was detected in the hatching rates of eggs from Experiment II $\left.\left(\mathrm{F}_{(3,40}\right)=4.221, p=0.011\right)$, with median hatching rates of $0.13,0.30,0.06$ and 0.64 for eggs from females
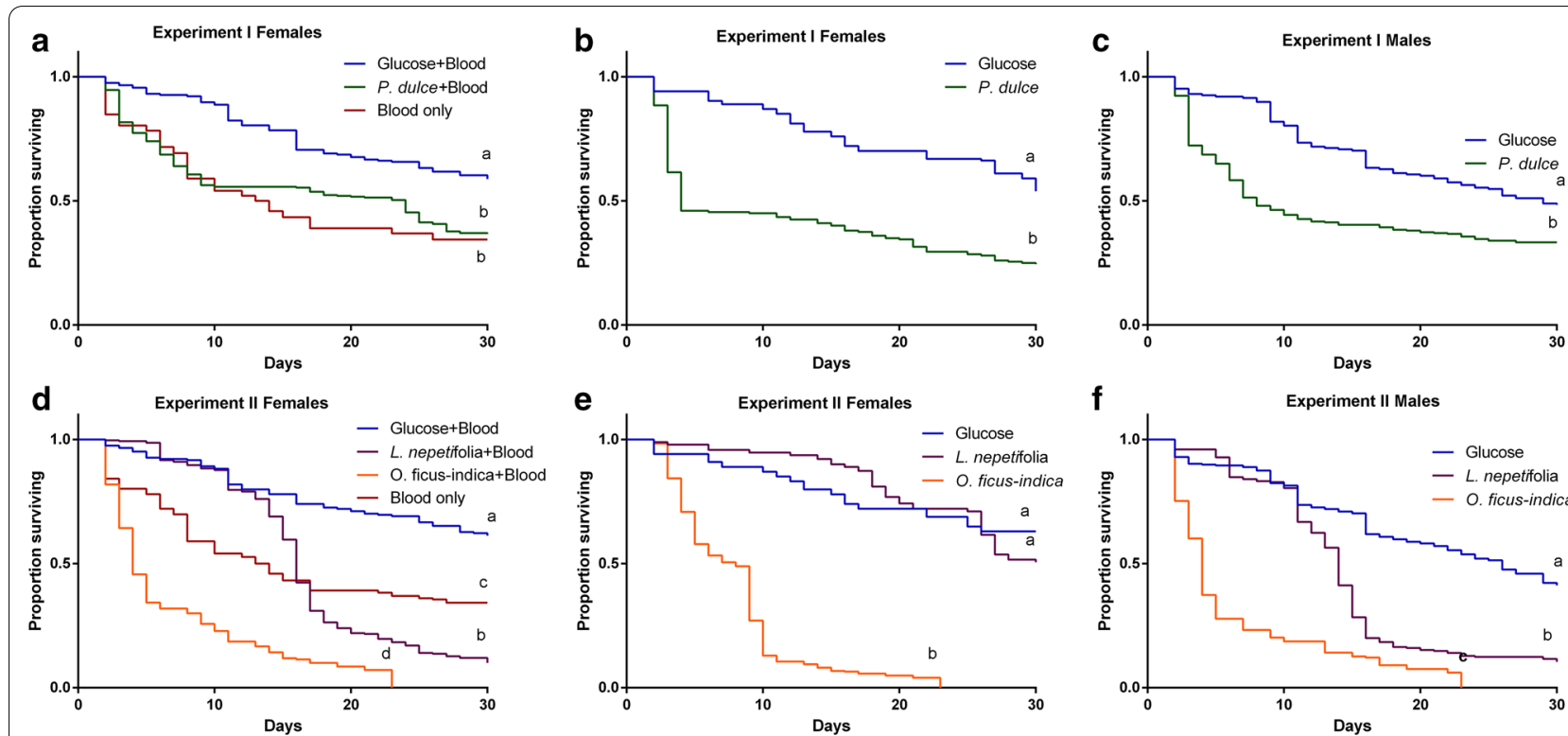

Fig. 2 The impact of plant nutrients on Ae. aegypti male and female survival. a Survival curves of female Ae. aegypti on P. dulce, 6\% glucose solution and exclusively mouse blood, with an initial three blood meal rations offered to those held on $P$. dulce and glucose solution $(P$-value $<0.001)$. (b-c) Survival curves of female and male Ae.aegypti, respectively, fed on $P$. dulce and glucose without initial blood meal $(P$-value $<0.001)$. d Survival curves of females held on L. nepetifolia, O. ficus-indica, $6 \%$ glucose solution and exclusively mouse blood, with the former three offered three initial blood meal rations ( $P$-value $<0.001)$. (e-f) Survival curves of female and male Ae. aegypti, respectively, fed on L. nepetifolia, $O$. ficus-indica or glucose solution without an initial blood meal $(P$-value $<0.001)$. Survival curves denoted by different letters are significantly different. Differences in survival curves were detected using Kaplan-Meier analysis and Cox regression analyses 


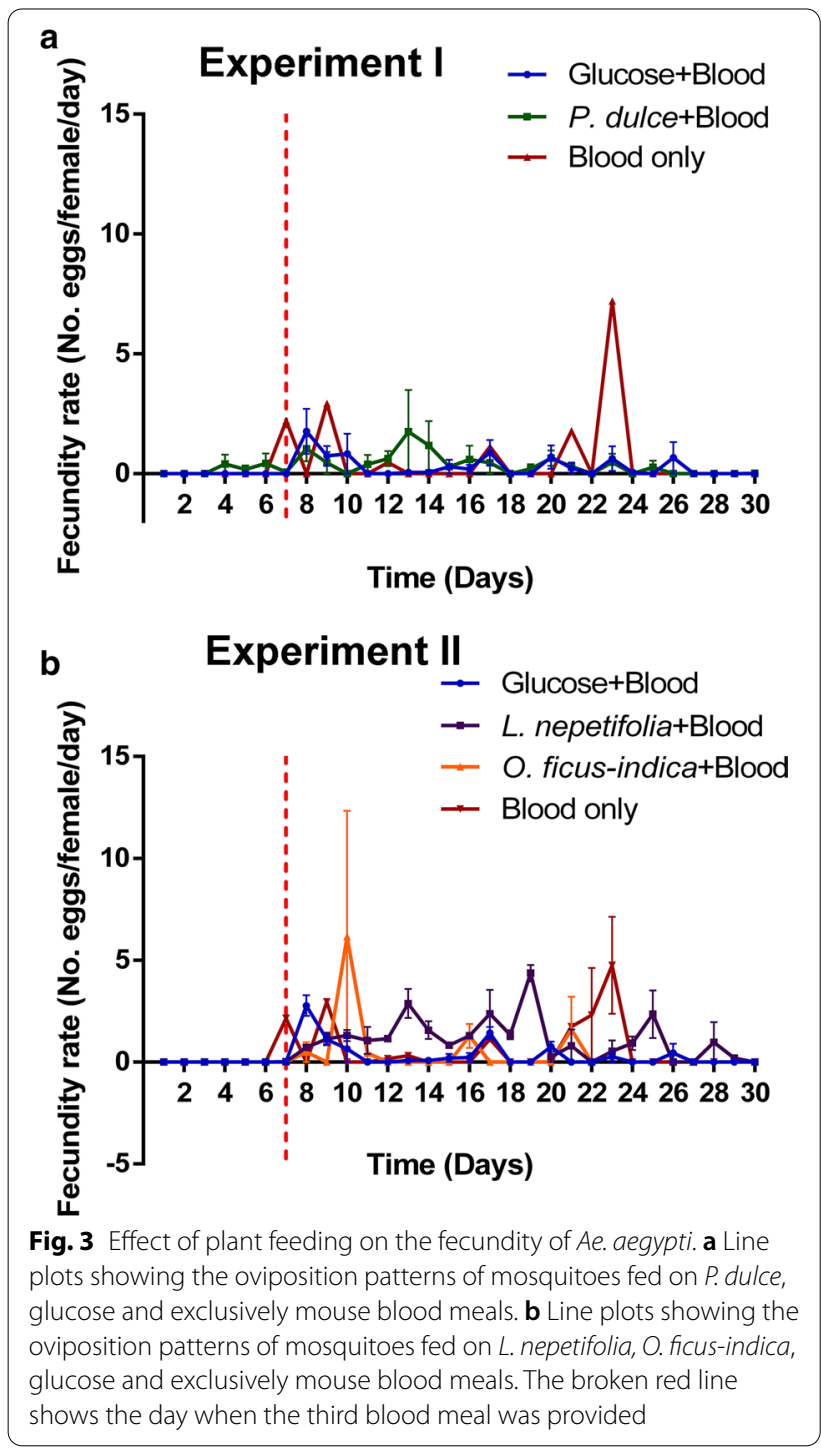

fed on glucose, L. nepetifolia, O. ficus-indica and blood meal, respectively (Fig. 4b).

\section{Variable nutrient quality support: observed differences} in the fitness matrix of Ae. aegypti fed on different host plants

A total of 12 amino acids present in mouse blood were detected in variable amounts in the sap plus nectar of three plant species. These included valine, serine, glutamine, proline, glycine, methionine, tyrosine, isoleucine, leucine, phenylalanine, tryptophan and arginine. Uniquely abundant amino acids detected in the guts of mosquitoes fed on the nutrient regimes included valine, arginine, isoleucine, methionine and phenylalanine (Fig. 5a). Valine was 5-, 13- and 14-fold more abundant in the guts of mosquitoes fed on L. nepetifolia, glucose

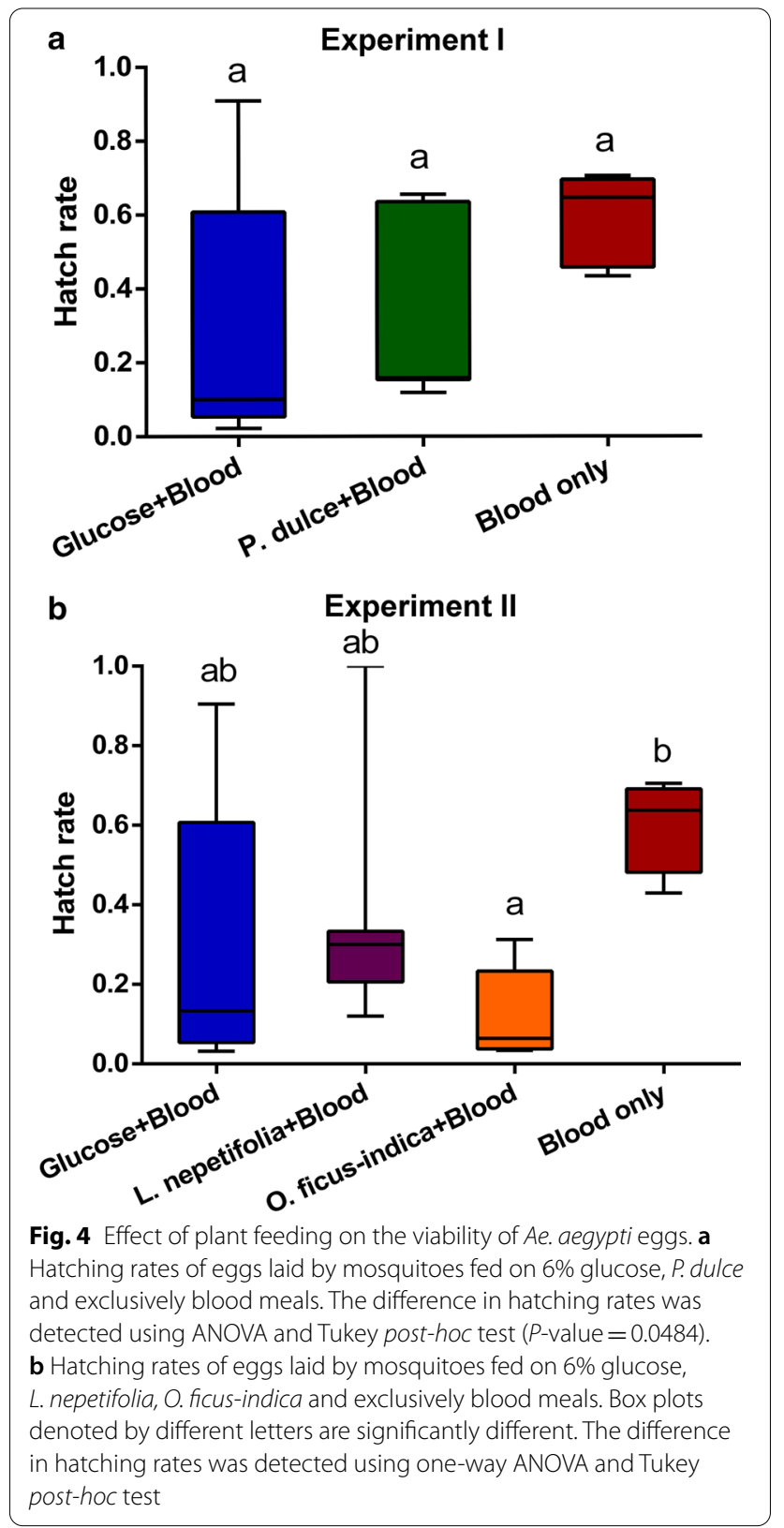

and O. ficus-indica, respectively. In addition, arginine was abundant in the guts of those fed on O. ficus-indica, isoleucine in those fed on $P$. dulce and methionine in those fed on L. nepetifolia (Fig. 5b). On the other hand, phenylalanine was 6-, 8- and 11-fold less abundant in the guts of mosquitoes fed on P. dulce, O. ficus-indica and glucose solution, respectively, relative to those exclusively fed on blood (Fig. 5b). Notably, glutamic acid was present in the guts of mosquitoes fed on mouse blood but absent in those from all the other diets. To further confirm that female Ae. aegypti indeed were able to imbibe these amino acids from their host plants, we analyzed four of 

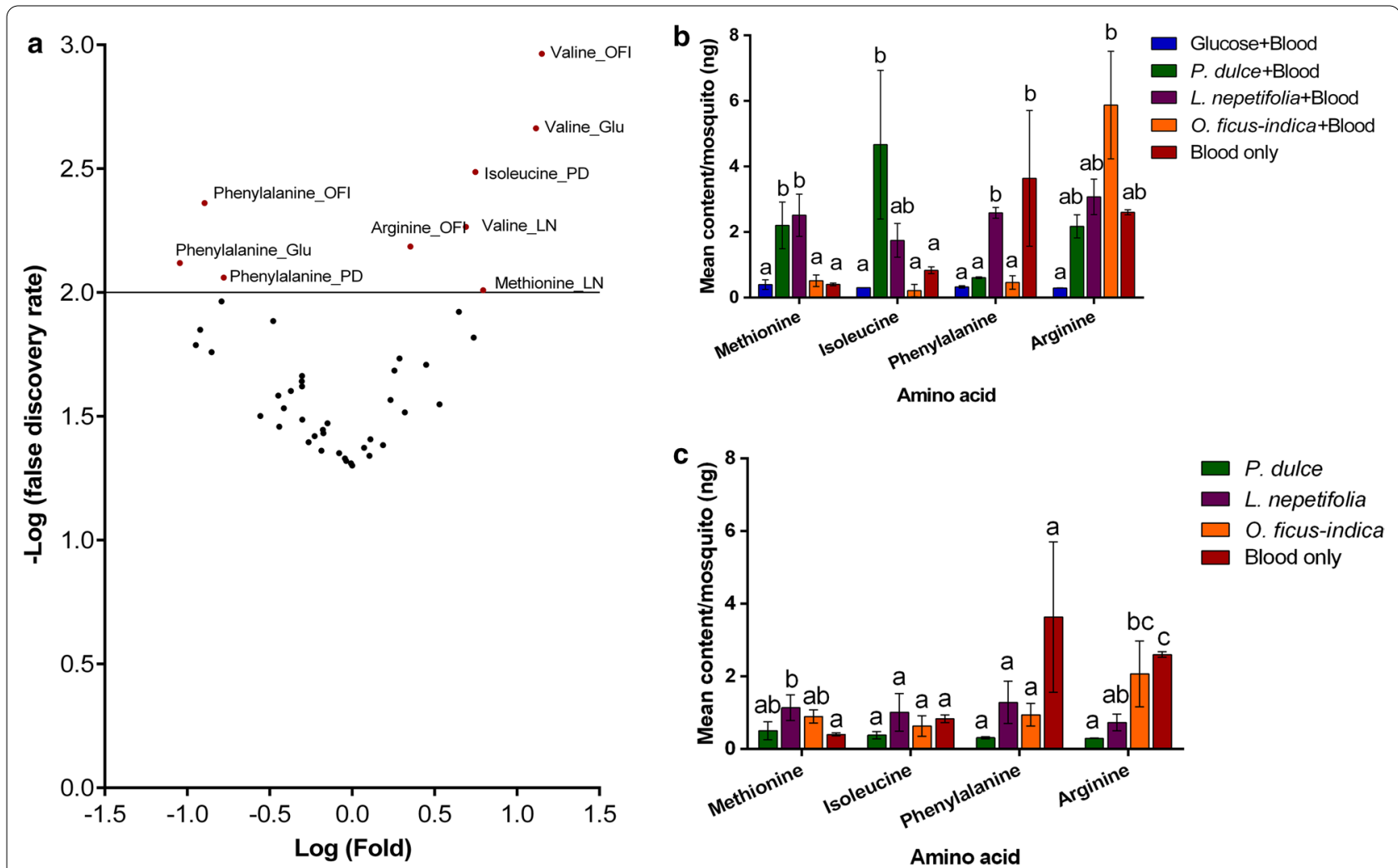

Fig. 5 Amino acid content of gut of Ae. aegypti fed of different nutrient sources. a Volcano plot depicting unique amino acids significantly abundant or low in the guts of females fed on P. dulce, L. nepetifolia, O. ficus-indica and $6 \%$ glucose solution compared to those exclusively fed on mouse blood. The horizontal line shows where $P=0.05$ by $F$ statistics. The significantly high or low abundant amino acids are shown. $L N$ denotes $L$. nepetifolia, PD denotes $P$. dulce, OFI denotes $O$. ficus-indica, Glu denotes glucose solution. $\mathbf{b}$ Mean amounts of the unique amino acids in the guts of females fed on the five nutrient sources with three initial blood meals. The $P$-values were $<0.001,<0.01,<0.05$ and $<0.01$ for methionine, isoleucine, phenylalanine and arginine, respectively. $\mathbf{c}$ Mean amounts of the unique amino acids in the guts of females fed on the three plant nutrients with no initial blood meals and those fed exclusively on blood. The $P$-values were $<0.05,=0.25,=0.052$ and $<0.01$ for methionine, isoleucine, phenylalanine and arginine, respectively. The differences in gut amino acid content were detected by one-way ANOVA and Tukey post-hoc test. Bars denoted by different letters are significantly different

the identified amino acids in the guts of non-blood-fed mosquitoes. Besides valine, none of the females from the glucose diet group had any detectable amino acids in their guts. However, females fed on all three host plants had variable amounts of methionine, isoleucine, phenylalanine and arginine in their guts (Fig. 5c).

No significant differences were detected in the amount of sugar ingested by male Ae. aegypti $\left(\mathrm{F}_{(3,93}\right)=1.389$, $P=0.251$; Additional file 1: Fig. S1). However, females fed on L. nepetifolia imbibed significantly more sugar compared to those fed on O. ficus-indica $\left(\mathrm{F}_{(3,93}\right)=2.791$, $P=0.0449$; Additional file 1: Fig. S1).

\section{Discussion}

Our findings show that the three plants used in this study differentially impact the survival and reproductive fitness of dengue vector, Ae. aegypti. We previously reported a sugar feeding frequency of $17 \%$ in female Ae. aegypti collected around vegetations in the coastal area of Kenya [16]. The study by Olson et al. [17] demonstrated that sugar feeding occurs at a much higher frequency than previously reported, with collection method and season being important in influencing the proportion of fructose-positive females captured. Plant sugars, particularly fructose, have been shown to provide a ready source of energy for various metabolic processes in several mosquito species [17, 30-33]. Extended survival time is pivotal in the transmission of vector-borne diseases as it guarantees completion of extrinsic incubation of the causative agents and increases the chances of multiple infective vertebrate host bites [34]. These findings further reinforce the argument of the central role played by plants in the biology of Ae. aegypti, contrary to previous beliefs [19-22].

While no eggs were laid by mosquitoes exclusively fed on the three plant species, significant differences in 
fecundity were observed when mosquitoes fed on them with initial blood meal rations. Those fed L. nepetifolia had slightly higher oviposition than their exclusively blood-fed counterparts, while those fed on P. dulce, $O$. ficus-indica and glucose laid fewer eggs than bloodfed females. Similar impacts of plant diets on mosquito fecundity have been observed for An. gambiae [30, 35] and Culex pipiens [33]. Although sugar feeding has long been suggested to impact the fecundity of Aedes mosquitoes [36,37], to the best of our knowledge, this is the first evidence directly linking plant feeding to Ae. aegypti fecundity. Plant nectars have been shown to increase mating competence in males of different mosquito species [31, 32, 38, 39]. Sugar has also been shown to be important in inducing egg development in autogenous Ae. albopictus and Cx. pipiens $f$. molestus [40, 41]. The failure of Ae. aegypti to lay eggs without an initial blood meal in this study is not surprising, although varying degrees of autogeny has been reported among these species in East Africa [42, 43]. However, the potential of $L$. nepetifolia to not only boost their overall fecundity but also induce a sustained oviposition long after the last blood meal is noteworthy. Although lower than the hatching rates in eggs from exclusively blood-fed females, a good proportion of eggs laid by females fed on L. nepetifolia were viable. The difference in fecundity of mosquitoes held on different nutrient sources observed in this study can be explained by three propositions: (i) males fed on L. nepetifolia had sugar-rich diets and therefore increased mating competence and reproductive output in females compared to males from the exclusively blood meal diet, which died off within 3 days; (ii) females held on L. nepetifolia imbibed sufficient sugar meals/ sap from the succulent plant tissues, thereby resulting in constant distention of the abdomen and inducing oocyte maturation following the initial blood meal, as has been reported for Ae. albopictus [40]; (iii) mosquitoes feeding on the three plants, especially on L. nepetifolia, imbibed not only sugar but also amino acids, which supplemented those received from the initial blood meal, further boosting their reproduction.

We explored the third proposition further by analyzing the amino acid content of female Ae. aegypti held on these plant species and comparing the outputs with those from mosquitoes fed exclusively on blood and their plant sources. Twelve amino acids present in mouse blood were positively identified in the plant sap/nectar and the guts of blood-fed females held on different diets in varying proportions. Notably, mosquitoes held on L. nepetifolia had high methionine content in their guts, while those held on $P$. dulce had high isoleucine content. Intriguingly, phenylalanine was significantly low in females held on $P$. dulce, O. ficus-indica and glucose solution, with mosquitoes fed on the three plant species and glucose lacking glutamic acid. Non-blood-fed females held on the three plant species had similar amino acid profiles as those offered an initial blood meal. These observations support our proposition that female Ae. aegypti imbibed variable amounts of amino acids from these plant species, which differentially impacted their fecundity. This was further supported by the detection of these amino acids in the respective host plants on which the mosquitoes were held. Different amino acids have been shown to impact differently on mosquito fecundity. Phenylalanine and tyrosine have been shown to be important for the development and tanning of An. gambiae and Ae. aegypti eggs $[44,45]$, while isoleucine is important in follicular maturation and preventing egg resorption in Ae. aegypti [46]. Methionine and leucine have been shown to increase the fecundity of green pea aphid, Cyrthosiphon pisum by enhancing the target of rapamycin (TOR) signaling pathway [47]. This study represents the first empirical evidence of the possible involvement of plant-derived amino acids in the reproductive fitness of Ae. aegypti.

We acknowledge that the study has some limitations that warrant further consideration: (i) in this study, individual females that blood fed were not separated from those that did not blood feed; (ii) we did not include a control with sugar continuously available and a blood meal provided every other day to allow males to survive longer and improve their mating competence; (iii) the use of plant cuttings in the assays with $P$. dulce and whole potted plants in the case of L. nepetifolia and O. ficusindica could have contributed to the observed differences in the performance of Ae. aegypti. Although L. nepetifolia significantly improved the survival and fecundity of $A e$. aegypti, it was not identified as a natural host plant of this species in our previous study and is not found within the locality where their eggs were collected. However, it serves as an important pointer to the potential of other plants with similar nutritional value to impact mosquito vector populations.

\section{Conclusion}

We conclude that these findings offer significant insight into the role of plant-derived nutrients in the biology and population dynamics of Ae. aegypti. Our study provides empirical evidence that plant-derived amino acids such as phenylalanine, methionine, isoleucine and arginine could play a role in sustaining the reproductive fitness of these disease vectors in situations where vertebrate hosts are scarce and only a few blood meal encounters are possible. 


\section{Supplementary information}

The online version contains supplementary material available at https://doi. org/10.1186/s13071-020-04519-y.

Additional file 1. Methods and results of Aedes aegypti gut sugar content analysis.

\section{Acknowledgements}

The authors thank Dr. Charles Mbogo and Dr. Joseph Mwangangi of KEMRIWellcome Trust Kilifi for their logistical support and Onesmus B. Wanyama and Xavier Cheseto for technical support.

\section{Authors' contributions}

Conceived and designed the experiments: VON, DPT, CLS, CP, BT. Performed the experiments: VON, DPT, MNM, BT. Analyzed the data: VON, DPT, BT. Wrote the paper: VON, DPT, CLS, CP, BT. All authors approved the final version for submission. All authors read and approved the final manuscript.

\section{Funding}

This study was funded by the Swedish International Development Cooperation Agency (Sida) studentship to VON and for local support at UP by the NRF awarded to CLS and CWWP. We also acknowledge the financial support by icipe's core donors, the UK's Foreign, Commonwealth and Development Office (FCDO), the Swedish International Development Cooperation Agency (Sida), the Swiss Agency for Development and Cooperation (SDC), Federal Democratic Republic of Ethiopia and the Kenyan Government.

\section{Availability of data and materials}

The datasets used and/or analysed during the current study are available from the corresponding author on reasonable request.

\section{Ethical approval and consent to participate}

Mice (BALB/c strain) used for mosquito blood feeding in these experiments were supplied by icipe Animal Rearing and Containment Unit. All blood-feeding experiments were conducted according to IACUC-approved protocols. Approval for the study was sought from the Kenya Medical Research Institute Scientific and Ethics Review Unit (KEMRI-SERU) (project no. SERU 2787).

\section{Consent for Publication}

Not applicable.

\section{Competing interests}

The authors declare that they have no competing interest.

\section{Author details}

${ }^{1}$ International Centre of Insect Physiology and Ecology (Icipe), Nairobi, Kenya. ${ }^{2}$ Department of Bioscience, Kenya Medical Research Institute-Wellcome Trust, Kilifi, Kenya. ${ }^{3}$ Department of Zoology and Entomology, University of Pretoria, Hatfield, South Africa.

Received: 22 July 2020 Accepted: 4 December 2020

Published online: 04 January 2021

\section{References}

1. Jaensich T, Junghanss T, Wills B, Brady OJ, Eckerle I, Farlow A, et al. Dengue expansion in Africa-not recognized or not happening? Emerg Infect Dis. 2014;20:e140487.

2. Guo C, Zhou Z, Wen Z, Liu Y, Zeng C, Xiao D, et al. Global epidemiology of dengue outbreaks in 1990-2015: a systematic review and meta-analysis. Front Cell Infect Microbiol. 2017;7:317.

3. World Health Organization. Dengue and severe dengue. World Health Organization. 2020 [cited 2020 May 21]. https://www.who.int/news$\mathrm{room} /$ fact-sheets/detail/dengue-and-severe-dengue.

4. Caron M, Grard G, Paupy C, Mombo IM, Bikie Bi Nso B, Kassa Kassa FR, et al. First evidence of simultaneous circulation of three different dengue virus serotypes in Africa. PLoS ONE. 2013;8:e78030.
5. Guzman MG, Harris E. Dengue. Lancet. 2015;385:453-65.

6. Attar N. ZIKA virus circulates in new regions. Nat Rev Microbiol. 2016;14:62-62.

7. Fauci AS, Morens DM. Zika virus in the Americas-Yet another arbovirus threat. N Engl J Med. 2016:374:601-4.

8. Dick GWA, Kitchen SF, Haddow AJ. Zika Virus (I). Isolations and serological specificity. Trans Royal Soc Trop Med Hyg. 1952;46:509-20.

9. Petersen LR, Jamieson DJ, Powers AM, Honein MA. Zika Virus. N Engl J Med. 2016:374:1552-63.

10. Benelli G, Mehlhorn H. Declining malaria, rising of dengue and Zika virus: insights for mosquito vector control. Parasitol Res. 2016;115:1747-54.

11. Murray NEA, Quam MB, Wilder-Smith A. Epidemiology of dengue: past, present and future prospects. CLEP. 2013;5:299-309.

12. Kraemer MU, Sinka ME, Duda KA, Mylne AQ, Shearer FM, Barker CM, et al. The global distribution of the arbovirus vectors Aedes aegypti and Ae. albopictus. eLIFE. 2015:4:e08347.

13. Ebi KL, Nealon J. Dengue in a changing climate. Environ Res. 2016:151:115-23.

14. Foster WA. Mosquito sugar feeding and reproductive energetics. Annu Rev Entomol. 1995:40:443-74.

15. Nyasembe VO, Torto B. Volatile phytochemicals as mosquito semiochemicals. Phytochem Lett. 2014;8:196-201.

16. Nyasembe VO, Tchouassi DP, Pirk CWW, Sole CL, Torto B. Host plant forensics and olfactory-based detection in Afro-tropical mosquito disease vectors. PLoS Negl Trop Dis. 2018;12:e0006185.

17. Olson MF, Garcia-Luna S, Juarez JG, Martin E, Harrington LC, Eubanks MD, et al. Sugar feeding patterns for Aedes aegypti and Culex quinquefasciatus (Diptera: Culicidae) mosquitoes in South Texas. J Med Entomol. 2020;1-9.

18. Graca-Souza A, Maya-Monteiro C, Paivasilva G, Braz G, Paes M, Sorgine $\mathrm{M}$, et al. Adaptations against heme toxicity in blood-feeding arthropods. Insect Biochem Mol Biol. 2006:36:322-35.

19. Scott TW, Naksathit A, Day JF, Kittayapong P, Edman JD. A fitness advantage for Aedes aegypti and the viruses it transmits when females feed only on human blood. Amer J Trop Med Hyg. 1997;57:235-9.

20. Costero A, Edman JD, Clark GG, Scott TW. Life table study of Aedes aegypti (Diptera: culicidae) in Puerto Rico fed only human blood versus blood plus sugar. J Med Entomol. 1998;35:809-13.

21. Naksathit AT, Edman JD, Scott TW. Partitioning of glycogen, lipid, and sugar in ovaries and body remnants of female Aedes aegypti (Diptera: culicidae) fed human blood. J Med Entomol. 1999:36:18-22.

22. Harrington LC, Edman JD, Scott TW. Why do female Aedes aegypti (Diptera: culicidae) feed preferentially and frequently on human blood? J Med Entomol. 2001;38:411-22.

23. Junnila A, Müller GC, Schlein Y. Species identification of plant tissues from the gut of An. sergentii by DNA analysis. Acta Tropica. 2010;1 15:227-33.

24. Jurado-Rivera JA, Vogler AP, Reid CAM, Petitpierre E, Gómez-Zurita J. DNA barcoding insect - host plant associations. Proc R Soc B. 2009;276:639-48.

25. Jinbo U, Kato T, Ito M. Current progress in DNA barcoding and future implications for entomology: DNA barcoding for entomology. Entomol Sci. 2011;14:107-24

26. Miller MA, Müller GC, Kravchenko VD, Vernon KK, Matheson CD, Hausmann A. DNA-based identification of Lepidoptera larvae and plant meals from their gut contents. Russian Entomol J. 2006;15:427-32.

27. Abbasi I, Trancoso Lopo de Queiroz A, Kirstein OD, Nasereddin A, Horwitz BZ, Hailu A, et al. Plant-feeding phlebotomine sand flies, vectors of leishmaniasis, prefer Cannabis sativa. Proc Natl Acad Sci USA. 2018:115:11790-5.

28. Özcan S, Şenyuva HZ. Improved and simplified liquid chromatography/ atmospheric pressure chemical ionization mass spectrometry method for the analysis of underivatized free amino acids in various foods. J Chromatogr A. 2006;1135:179-85

29. R Core Team. R: A language and environment for statistical computing [Internet]. Vienna, Austria: R Foundation for Statistical Computing; 2020. Available from:https://www.R-project.org/.

30. Manda H, Gouagna LC, Foster WA, Jackson RR, Beier JC, Githure Jl, et al. Effect of discriminative plant-sugar feeding on the survival and fecundity of Anopheles gambiae. Malar J. 2007;6:113.

31. Gary RE, Cannon JW, Foster WA. Effect of sugar on male Anopheles gambiae mating performance, as modified by temperature, space, and body size. Parasites Vectors. 2009;2:19. 
32. Bellini R, Puggioli A, Balestrino F, Brunelli P, Medici A, Urbanelli S, et al. Sugar administration to newly emerged Aedes albopictus males increases their survival probability and mating performance. Acta Trop. 2014;132:S116-23.

33. Yu B-T, Ding Y-M, Mo X-C, Liu N, Li H-J, Mo J-C. Survivorship and fecundity of Culex pipiens pallens feeding on flowering plants and seed pods with differential preferences. Acta Trop. 2016;155:51-7.

34. Vaidyanathan R, Fleisher AE, Minnick SL, Simmons KA, Scott TW. Nutritional stress affects mosquito survival and vector competence for West Nile virus. Vector-Borne Zoonotic Diseases. 2008:8:727-32.

35. Impoinvil DE, Kongere JO, Foster WA, Njiru BN, Killeen GF, Githure Jl, et al. Feeding and survival of the malaria vector Anopheles gambiae on plants growing in Kenya. Med Vet Entomol. 2004;18:108-15.

36. Nayar JK, Sauerman Jr DM. The Effects of nutrition on survival and fecundity in Florida mosquitoes Part 3. Utilization of blood and sugar for fecundity1. J Med Entomol. 1975;12:220-5.

37. Anderson HI. The effect of sugar meals and body size on fecundity and longevity of female Aedes communis (Diptera: culicidae). Physiol Entomol. 1992;17:203-7.

38. Gouagna L-C, Poueme RS, Dabiré KR, Ouédraogo J-B, Fontenille D, Simard F. Patterns of sugar feeding and host plant preferences in adult males of Anopheles gambiae (Diptera: culicidae). J Vect Ecol. 2010;35:267-76.

39. Chadee DD, Sutherland JM, Gilles JRL. Diel sugar feeding and reproductive behaviours of Aedes aegypti mosquitoes in Trinidad: with implications for mass release of sterile mosquitoes. Acta Trop. 2014;132:S86-90.

40. Chambers GM, Klowden MJ. Distention and sugar feeding induce autogenous egg development by the Asian tiger mosquito (Diptera: culicidae). J Med Entomol. 1996;33:372-8.
41. Kassim NFA, Webb CE, Russell RC. Is the expression of autogeny by Culex molestus Forskal (Diptera: culicidae) influenced by larval nutrition or by adult mating, sugar feeding, or blood feeding? J Vect Ecol. 2012;37:162-71.

42. Trpis M. Autogeny in diverse populations of Aedes aegypti from East Africa. Tropenmedizin und Parasitologie. 1977;28:77-82.

43. Ariani CV, Smith SCL, Osei-Poku J, Short K, Juneja P, Jiggins FM. Environmental and genetic factors determine whether the mosquito Aedes aegypti lays eggs without a blood meal. Amer J Trop Med Hyg. 2015.715-21.

44. Li J, Christensen BM. Involvement of L-tyrosine and phenol oxidase in the tanning of Aedes aegypti eggs. Insect Biochem Mol Biol. 1993;23:739-48.

45. Fuchs S, Behrends V, Bundy JG, Crisanti A, Nolan T. Phenylalanine metabolism regulates reproduction and parasite melanization in the malaria mosquito. PLoS ONE. 2014;9:e84865.

46. Chang Y-YH, Judson CL. The role of isoleucine in differential egg production by the mosquito Aedes aegypti Linnaeus (Diptera: Culicidae) following feeding on human or guinea pig blood. Comp Biochem Physiol Part A: Physiol. 1977;57:23-8.

47. Gao J, Guo H, Sun Y, Ge F. Differential accumulation of leucine and methionine in red and green pea aphids leads to different fecundity in response to nitrogen fertilization. Pest Manag Sci. 2018;74:1779-89.

\section{Publisher's Note}

Springer Nature remains neutral with regard to jurisdictional claims in published maps and institutional affiliations.
Ready to submit your research? Choose BMC and benefit from:

- fast, convenient online submission

- thorough peer review by experienced researchers in your field

- rapid publication on acceptance

- support for research data, including large and complex data types

- gold Open Access which fosters wider collaboration and increased citations

- maximum visibility for your research: over 100M website views per year

At BMC, research is always in progress.

Learn more biomedcentral.com/submissions 